\title{
Towards a compact atomic clock based on coherent population trapping and the grating magneto-optical trap
}

\author{
Gregory W. Hoth, Rachel Elvin, Michael Wright, Ben Lewis, Aidan S. Arnold, Paul F. Griffin, \\ and Erling Riis ${ }^{\mathrm{a}}$ \\ aDept. of Physics, SUPA, University of Strathclyde, Glasgow, G4 0NG, UK
}

\begin{abstract}
The combination of coherent population trapping $(\mathrm{CPT})$ and laser cooled atoms is a promising platform for realizing the next generation of compact atomic frequency references. Towards this goal, we have developed an apparatus based on the grating magneto-optical trap (GMOT) and the high-contrast lin $\perp$ lin CPT scheme in order to explore the performance that can be achieved. One important trade-off for cold-atom systems arises from the need to simultaneously maximize the number of cold atoms available for interrogation and the repetition rate of the system. This compromise can be mitigated by recapturing cold atoms from cycle to cycle. Here, we report a quantitative characterization of the cold atom number in the recapture regime for our system, which will enable us to optimize this trade-off. We also report recent measurements of the short-term frequency stability with a short-term Allan deviation of $3 \times 10^{-11} / \sqrt{\tau}$ up to an averaging time of $\tau=10 \mathrm{~s}$.
\end{abstract}

Keywords: Coherent Population Trapping. Grating Magneto-Optical Trap. Microwave Atomic Clock. Laser Cooling. Cold-Atoms. Micro-fabricated Grating Chips.

\section{INTRODUCTION}

After many years of development, atomic clocks based on laser cooled atoms are beginning to be made commercially. ${ }^{1-3}$ These commercial cold-atom clocks utilize microwave interrogation of the sample and achieve a short-term fractional frequency stability on the order of $10^{-13} / \sqrt{\tau}$ with the ability to average down to the level of $3 \times 10^{-15}$ or better. The last two decades have also seen tremendous developments in atomic clocks based on optical interrogation of microwave atomic resonances via the coherent population trapping (CPT) technique., ${ }^{4,5}$ Vapor cell clocks based on high contrast $\mathrm{CPT}^{6-8}$ have also recently achieved short term frequency stabilities of a few times $10^{-13} / \sqrt{\tau}$ with long term behavior dominated by frequency drifts. ${ }^{6-9}$ In contrast to cold-atom microwave clocks and vapor cell CPT clocks, systems that combine laser cooling and CPT interrogation have been developed and studied relatively recently. ${ }^{9-16}$ The best of these cold-atom CPT systems has achieved a short-term frequency stability of $1.3 \times 10^{-11} / \sqrt{\tau}$, with the capability to average down to $1.8 \times 10^{-13} \cdot{ }^{13}$ So far, the short-term frequency stability of these cold-atom CPT systems has been mainly limited by technical noise, and estimates based on the quantum projection noise limit ${ }^{17,18}$ indicate that the short-term performance could be improved by more than a factor of ten. ${ }^{9,11}$ It is reasonable to imagine that the combination of cold atoms and CPT might provide a route to realizing an atomic clock with a short-term frequency stability on the order of $10^{-13} / \sqrt{\tau}$, good long-term stability, and a compact physics package. ${ }^{11,19}$ However, there is still much work to be done on the trade-offs in size and performance with different approaches to realizing compact cold-atom systems.

One promising approach for reducing the size and complexity of cold-atom systems is the grating magnetooptical trap ${ }^{20,21}$ (GMOT). In the GMOT, the three pairs of counter-propagating beams from a standard vapor cell $\mathrm{MOT}^{22}$ are replaced by a single beam incident on a micro-fabricated diffraction grating, which generates the remaining beams required to realize the MOT. Working towards the long-term goal of building a mobile cold-atom CPT system, we have built an apparatus ${ }^{15,16}$ to study the performance that can be achieved with lin $\perp$ lin $\mathrm{CPT}^{6,23,24}$ and the GMOT. In this presentation, we will focus on recent improvements to our apparatus that have allowed us to reach a short-term frequency stability of $3 \times 10^{-11} / \sqrt{\tau}$ for $\tau<10 \mathrm{~s}$. In section 2 , we

photonics.phys.strath.ac.uk/atom-optics 
quantify the trade-off in cycle time and atom number in the recapture regime ${ }^{25}$ for a GMOT based on three linear diffraction gratings. In section 3, we summarize our apparatus and recent upgrades that have improved the signal-to-noise ratio. In section 4, we report recent measurements of the frequency stability of our apparatus. Finally, section 5 concludes the paper with a discussion of future directions for our GMOT CPT clock.

\section{RECAPTURE WITH THE GRATING MAGNETO-OPTICAL TRAP}

For compact cold-atom systems, it is important to maximize the number of cold atoms while minimizing the sequence dead time. ${ }^{26-28}$ In many cases, this trade-off can be mitigated by recapturing the cold-atoms from cycle to cycle. ${ }^{11,25,29}$ Here, we characterize the efficiency of atom recapture with a grating magneto-optical trap (GMOT). We use a micro-fabricated chip formed of three linear diffraction gratings arranged with triangular symmetry. ${ }^{20,21}$ The chip is held outside a glass vacuum cell. The vacuum is maintained by a $2 \ell / \mathrm{s}$ ion pump. In order to laser cool ${ }^{87} \mathrm{Rb}$ atoms, we prepare light red detuned from the $|F=2\rangle \rightarrow\left|F^{\prime}=3\right\rangle$ transition, and light for hyperfine repumping, resonant with $|F=1\rangle \rightarrow\left|F^{\prime}=2\right\rangle$ transition. The light is fiber-coupled in a common single-mode optical fiber, collimated to a $1 / \mathrm{e}^{2}$ diameter of $20 \mathrm{~mm}$, circularly polarized with a quarter-waveplate, and the beam is directed to the grating chip. A pair of anti-Helmoltz coils, co-axial with the input beam and centred on the overlap volume, provide a quadrupole magnetic field with an axial gradient of $\sim 15 \mathrm{G} / \mathrm{cm}$.

During a typical experimental cycle, we load cold atoms into the GMOT for a time $T_{\mathrm{L}}$, then turn off the magnetic gradient and cool the atoms to sub-Doppler temperatures in an optical molasses. The final cloud temperature is $T \approx 40 \mu \mathrm{K}$, limited by a homogeneous bias magnetic field that is used to provide the quantization axis for the CPT experiment. After the molasses, the MOT light is turned off and the atoms fall freely for a time $T_{\mathrm{f}}$. Then, the MOT light is switched back on. As long as the cold atoms remain within the capture volume, they are quickly collected in the trap center. ${ }^{11,25,29}$ By operating in this recapture regime, one can obtain atom numbers comparable to $N_{\max }$, the maximum atom number in a continuously loaded MOT, with load times that are a fraction of the $1 /$ e MOT loading time.

In order to quantify the trade-off between repetition rate, interrogation time, and number of cold atoms, we have measured the steady state atom number as a function of the load time $T_{\mathrm{L}}$ and the free fall time $T_{\mathrm{f}}$. We compare our results to a model of the MOT in the recapture regime developed by Rakholia et al. ${ }^{25}$ By modeling the recapture process as a geometric sequence, Rakholia et al. ${ }^{25}$ show the expected steady-state atom number is given by

$$
N_{\mathrm{ss}}=N_{\max } \frac{T_{\mathrm{L}}}{T_{\mathrm{c}}} \frac{1}{1+\frac{1-r_{0}}{\beta T_{\mathrm{c}}}}
$$

Here, $N_{\max }$ is the maximum atom number when the trap is on continuously. $\beta$ is the rate at which cold atoms are lost, and $1 / \beta$ is the $1 / e$ MOT loading time. ${ }^{25}$ In typical operation, we measure loading time constants of $1 / \beta \approx 250 \mathrm{~ms} . T_{\mathrm{c}}$ is the total cycle time, given by the sum of the load time, the molasses duration, and the free fall time. Finally, $r_{0}$ is the recapture efficiency, defined as the fraction of cold atoms recaptured at the beginning of each cycle. ${ }^{25}$

We measured the recapture efficiency for our system as a function of the free fall time by loading a full MOT from vapor, applying optical molasses, releasing the atoms for a time $T_{\mathrm{f}}$, and then switching on the MOT. The recaptured fraction was estimated by comparing the fluorescence from the cold atoms just after the MOT was released to the fluorescence from the fully loaded MOT. The results are shown in Fig. 1a. For this study, we fit the measured recapture efficiency data empirically. In future work, it would be interesting to build a model of the recapture efficiency incorporating the geometry of the GMOT capture volume, the temperature of the cold atom cloud, and the spatial distribution of the atoms.

The other panels of Fig. 1 (b-d) illustrate the trade-off between the cycle parameters and the steady state atom number given the recapture efficiency in our apparatus. We find good agreement with the model developed by Rakholia et al. ${ }^{25}$ combined with our measured recapture efficiency. 

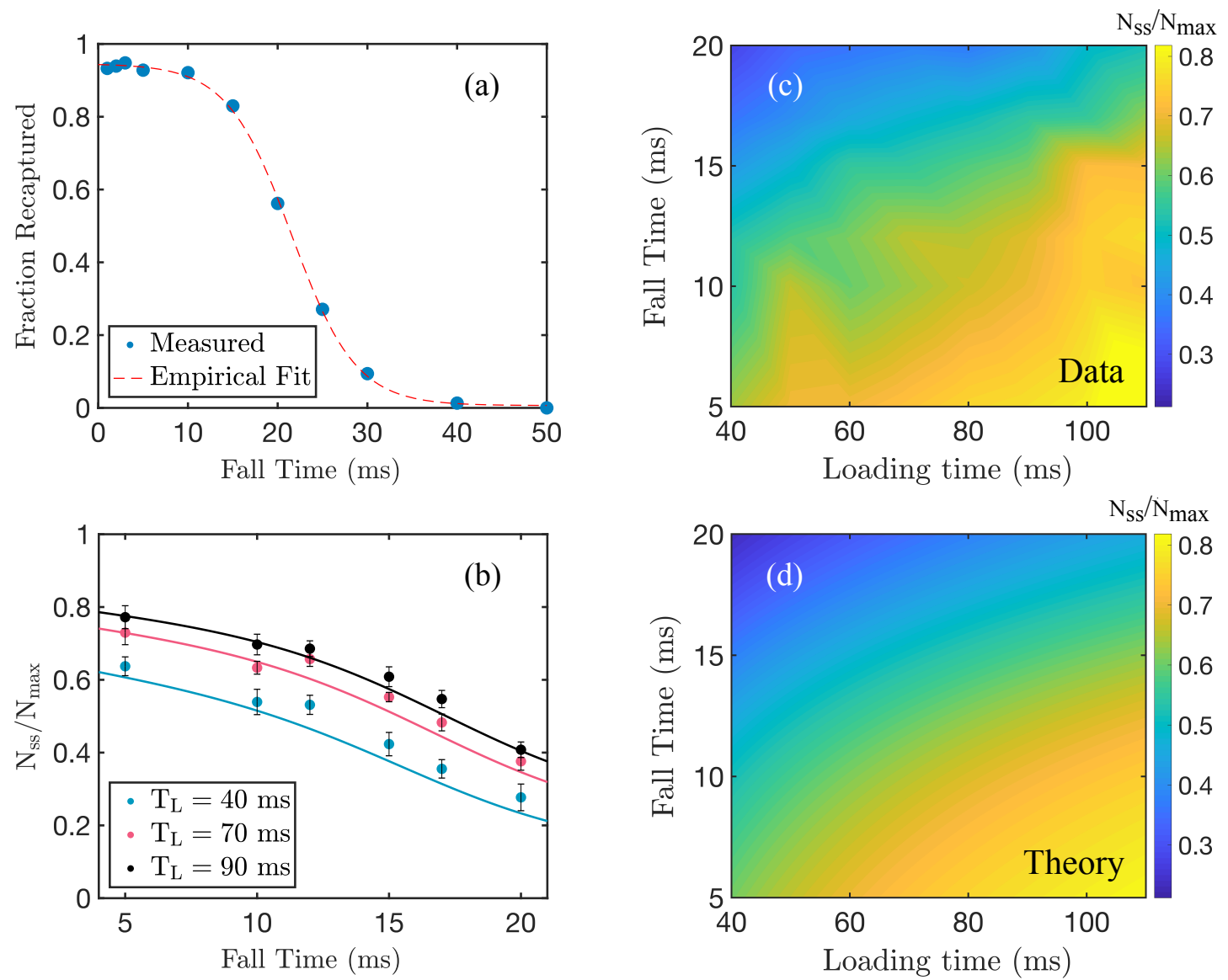

Figure 1. (a) Measurement of the fraction of atoms recaptured in the GMOT. Blue points are measured data whilst the red dotted line shows an empirical fit used to calculate the expected steady-state atom number. (b) Measurement of the steady-state atom number, $N_{\mathrm{ss}}$ achieved for different choices of loading and fall times, $T_{\mathrm{L}}$ and $T_{\mathrm{f}}$. (c-d) Measured data (c) and calculated values (d) for $N_{\mathrm{ss}}$ as a function of $T_{\mathrm{L}}$ and $T_{\mathrm{f}}$. Theoretical values are obtained through Eq. 1, where $r_{0}$ is given by the empirical fit. The steady state atom numbers are normalized to the maximum atom number measured with a long loading time.

\section{OPTICAL BENCH FOR LIN $\perp$ LIN CPT}

In this section, we briefly review the apparatus ${ }^{15,16}$ we are developing to investigate the performance that can be achieved in a cold atom CPT frequency standard based on the GMOT and the lin $\perp$ lin polarization scheme. ${ }^{6,23,24}$ We emphasize the addition of a phase locked loop (PLL) to our CPT apparatus, which has improved our short term frequency stability by a factor of two.

Figure 2 outlines the apparatus used to generate the light for addressing the CPT resonance, deliver it to the cold atoms, and detect the CPT signals. Light from a $795 \mathrm{~nm}$ ECDL locked to the $D_{1}$ line of ${ }^{87} \mathrm{Rb}$ is split into two optical paths. In one path, the light passes through an acousto-optical modulator (AOM 1 in Fig. 2) driven at frequency $f_{\mathrm{AOM}} \approx 200 \mathrm{MHz}$. In the other path, the light is modulated by a fiber-coupled electro-optical modulator $(\mathrm{EOM})$. We parameterize the frequency of the EOM driver as $f_{\mathrm{EOM}}=f_{\mathrm{hfs}}+f_{\mathrm{AOM}}+\Delta_{2, \mathrm{ph}}$ where $f_{\mathrm{hfs}} \approx 6.8 \mathrm{GHz}$ is the ground state hyperfine splitting and $\Delta_{2, \mathrm{ph}}$ is a frequency offset that we adjust to scan the $\mathrm{CPT}$ resonances. The EOM is driven so that the zeroth and \pm 1 orders have approximately equal powers. After these modulators, the two beams are recombined with a polarizing beam splitter (PBS).

By splitting the light from our ECDL into two paths, we are able to realize a pair of laser modes with orthogonal polarizations, good phase coherence, and a frequency difference of approximately $f_{\mathrm{hfs}}$ with a single laser, rather than a pair of phase locked lasers. ${ }^{6,23,24}$ The use of a single laser for lin $\perp$ lin CPT is attractive 


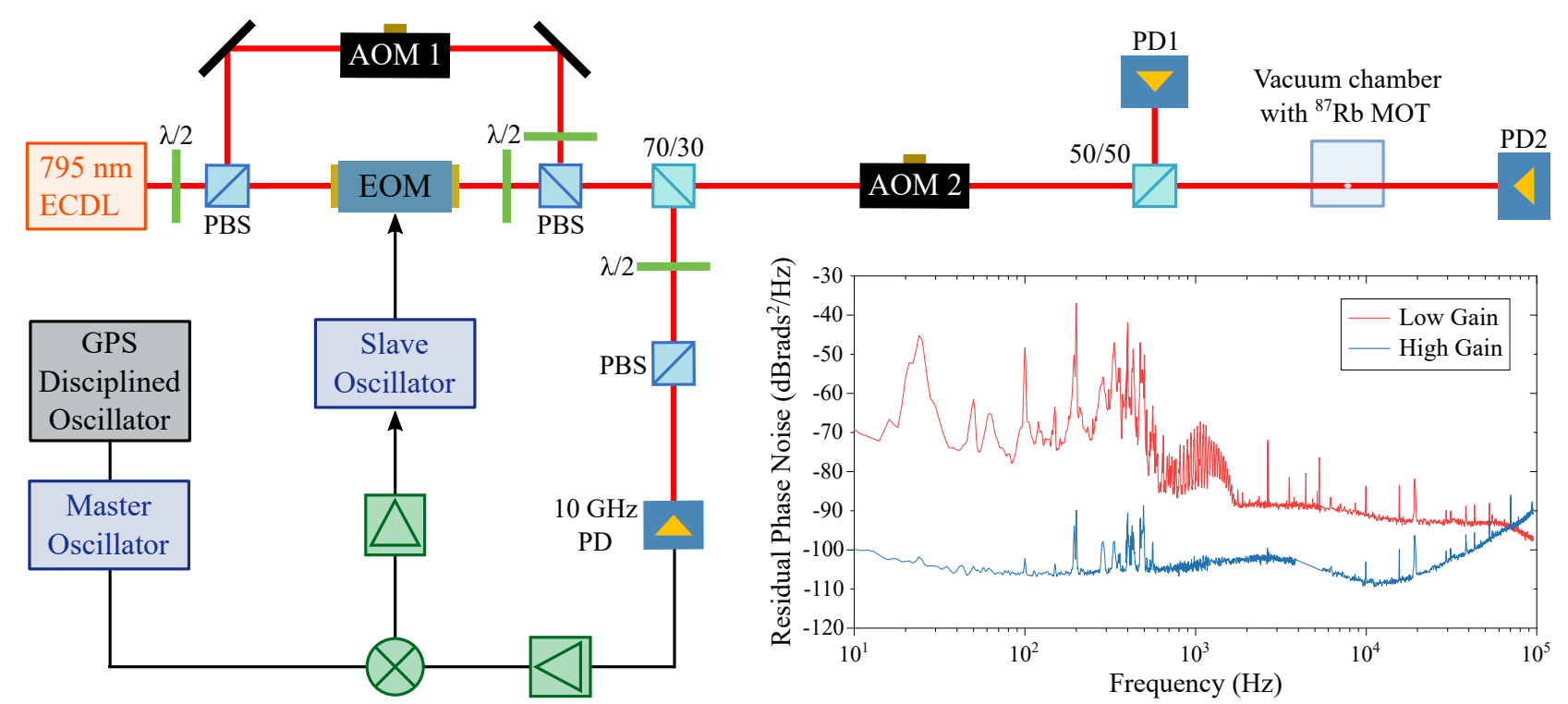

Figure 2. A schematic diagram of the optical system we have developed to realize lin $\perp$ lin $\mathrm{CPT}$ with cold ${ }^{87} \mathrm{Rb}$ atoms. The bottom left of the diagram shows the components of the PLL that we have added to stabilize the relative phase of the two CPT frequency components. The green symbols represent amplifiers and the mixer. (Inset) The Fourier spectrum of the mixer output with low (high) gain on the feedback to the EOM driver with the red (blue) traces. With the PLL weakly locked, one can see significant noise on the phase of the CPT beatnote. As the gain increases, this noise is suppressed and the CPT beatnote is phase locked to our reference oscillator.

for our long term goal of building a robust, compact device, but the optical system shown in Fig. 2 also comes with some drawbacks. In particular, fluctuations in the separated paths can cause noise on the relative phase of the two CPT components, and this phase noise can degrade the performance of CPT frequency standards..$^{9,12,30}$ Initial studies of the noise in our apparatus indicated that this optical phase noise was likely the dominant limitation to the short term frequency stability of our apparatus. Therefore, we have implemented a phase locked loop (PLL) to suppress the relative phase noise from the separated paths.

In order to implement the PLL, we split the combined beam using a non-polarizing beam splitter (NPBS)*. One output of the NPBS is detected with a fast photodiode while the other is sent to the cold atoms. With the fast photodiode, we measure the RF beatnotes produced by the interference of the beam modulated by AOM 1 and the beam modulated by the EOM. We use a mixer to compare the phase of the CPT beatnote to a reference signal from a commercial microwave synthesizer with frequency $f_{\text {master }}=f_{\mathrm{hfs}}+\Delta_{2, \mathrm{ph}}$. The output of the mixer is used to steer the frequency of the EOM driver, suppressing the phase noise of the CPT beatnote as shown in the inset to Fig. 2. In Sec. 4, we show the addition of the PLL also directly improves the short-term frequency stability of our apparatus.

After picking off some light to stabilize the relative phase of the CPT components with the PLL, we use the remainder of the light to interrogate the cold atoms. The combined beam passes through another AOM (AOM 2 in Fig. 2), which is used to generate pulses for the Ramsey CPT sequence. ${ }^{23}$ After the AOM, the beam is coupled into an optical fiber, and then directed through the cold atoms. Once all of the AOM frequency shifts are accounted for, including an AOM in the saturated absorption set up used to lock the ECDL (not shown in Fig. 2), the light incident on the atoms contains the two lin $\perp$ lin CPT frequency components, which are resonant with the $|F=1,2\rangle \rightarrow\left|F^{\prime}=2\right\rangle$ hyperfine transitions in the ${ }^{87} \mathrm{Rb} \mathrm{D}_{1}$ line. We use the $\left|F^{\prime}=2\right\rangle$ excited state in order to minimize the magnetic field sensitivity of the CPT Ramsey fringes as well as maximize the fringe amplitude. ${ }^{16,31}$ The CPT beam also contains several non-resonant frequency components. The zeroth

${ }^{*}$ It is also possible to obtain light for the PLL using the free port of the PBS which recombines the two beams, but we have found that the intensity and the polarization of the light delivered to the atoms is more stable if we use a NPBS to pick off light for the PLL after recombining the two independent paths. 
order component of the EOM output is of particular interest because it is detuned from the $|F=2\rangle$ to $\left|F^{\prime}=2\right\rangle$ transition by approximately $200 \mathrm{MHz}$. We plan to study the light shifts caused by the nearby sideband in future work. All the other frequency components are detuned by at least the hyperfine splitting.

A typical measurement sequence consists of loading the GMOT and then cooling the atoms in an optical molasses. The molasses is followed by a CPT Ramsey sequence consisting of a dark state pumping pulse, a free evolution period and a detection pulse. ${ }^{23}$ We measure CPT spectroscopy signals by scanning the frequency of our microwave synthesizer (PLL master in Fig. 2) and observing the transmission of the cold atoms. The CPT Ramsey fringe signal arises from the transient change in the transmission of the cold atoms at the beginning of the detection pulse. ${ }^{23}$ We measure the transmission of the cold atoms by using two photodiodes, ${ }^{9,11}$ one before and one after the beam interacts with the atoms (PD 1 and PD 2 in Fig. 2). With these two signals, it is possible to significantly suppress the influence of the intensity noise ${ }^{9,11}$ of the CPT beam on the detection. This normalisation process happens in two steps. ${ }^{9,11}$ The first step is to calculate the ratio of the two photodiode signals. Then, we take a second ratio to compare the integrated transmission at the beginning of the detection pulse to the integrated transmission after several multiples of the dark state-pumping time. With this double normalisation, we can suppress the laser intensity noise by a factor of 20 compared to the signal obtained from the beginning of the detection pulse with a single photodiode. This second normalisation can also be done by integrating the transmission at the end of the pumping pulse, ${ }^{9,11}$ but we obtain a small improvement in the intensity noise cancellation by normalising against the end of the detection pulse at the cost of a small increase in the duration of the measurement sequence. A typical $T=10 \mathrm{~ms}$ Ramsey fringe obtained in our apparatus is shown in the inset to Fig. 3.

\section{FREQUENCY STABILITY MEASUREMENTS}

To quantify the frequency stability of our apparatus, we ran the experiment sequence described in Sec. 3 repeatedly. For these measurements, we loaded cold atoms for about $70 \mathrm{~ms}$, and the Ramsey time was set to $T=10 \mathrm{~ms}$, corresponding to a detected Ramsey fringe pattern of width $\Delta f=50 \mathrm{~Hz}$. The steady state atom number was $N_{\mathrm{ss}} \approx 1 \times 10^{7}$, which is about $65 \%$ of the maximum atom number. The signal-to-noise ratio was approximately 40 in a single shot. During the measurement, the frequency of the PLL master oscillator alternated between the frequencies $f_{p} \pm \Delta f / 2$ where $f_{p}$ is the RF frequency corresponding to the peak of the central Ramsey fringe. This allowed us to measure the frequency difference between the mean oscillator output frequency and the atomic resonance frequency. The master PLL oscillator is referenced to a GPS disciplined oscillator, which has a specified fractional frequency stability of $<1 \times 10^{-12}$. Figure 3 shows the standard Allan deviation of these measured frequency differences taken with (grey circles) and without (red diamonds) the PLL operating.

With the PLL operating, the short-term stability is $\sigma_{y}(\tau)=3 \times 10^{-11} / \sqrt{\tau}$ for an observation time up to $10 \mathrm{~s}$, after which the frequency stability reaches a floor near $1 \times 10^{-11}$. This shows an obvious improvement to the stability without the PLL, which has a short-term stability of $\sigma_{y}(\tau)=6 \times 10^{-11} / \sqrt{\tau}$, and directly corresponds to improving the SNR on the side of the fringe from 20 to 40 by implementing the PLL.

Further investigation into the frequency stability limit near $1 \times 10^{-11}$ is currently underway. Since our system is unshielded, we operate with a relatively large bias field in order to ensure the quantization axis is well defined despite the presence of magnetic gradients. This means one likely source for the frequency instability is drift in the bias field. In order to probe the role of bias field fluctuations in the stability floor, we also measured the stability of our apparatus at a lower bias field value (Fig. 3 blue squares). As shown in Fig. 3, the data are consistent with a small improvement when reducing the magnetic bias field.

\section{CONCLUSION}

With the addition of the PLL to suppress the relative phase noise, our apparatus based on lin $\perp$ lin CPT and the GMOT has achieved a short-term frequency stability of $\sigma_{y}(\tau)=3 \times 10^{-11} / \sqrt{\tau}$. The recent improvements in the signal-to-noise ratio of our apparatus are encouraging, and we plan to continue to push towards the fundamental limit set by quantum projection noise. In particular, fluorescence detection is a promising approach to improve the signal-to-noise ratio. ${ }^{10}$ With our current measurement sequence, we estimate that a frequency stability ${ }^{17,18}$ of $\sigma_{y}(\tau)=2 \times 10^{-13} / \sqrt{\tau}$ could eventually be achieved. 


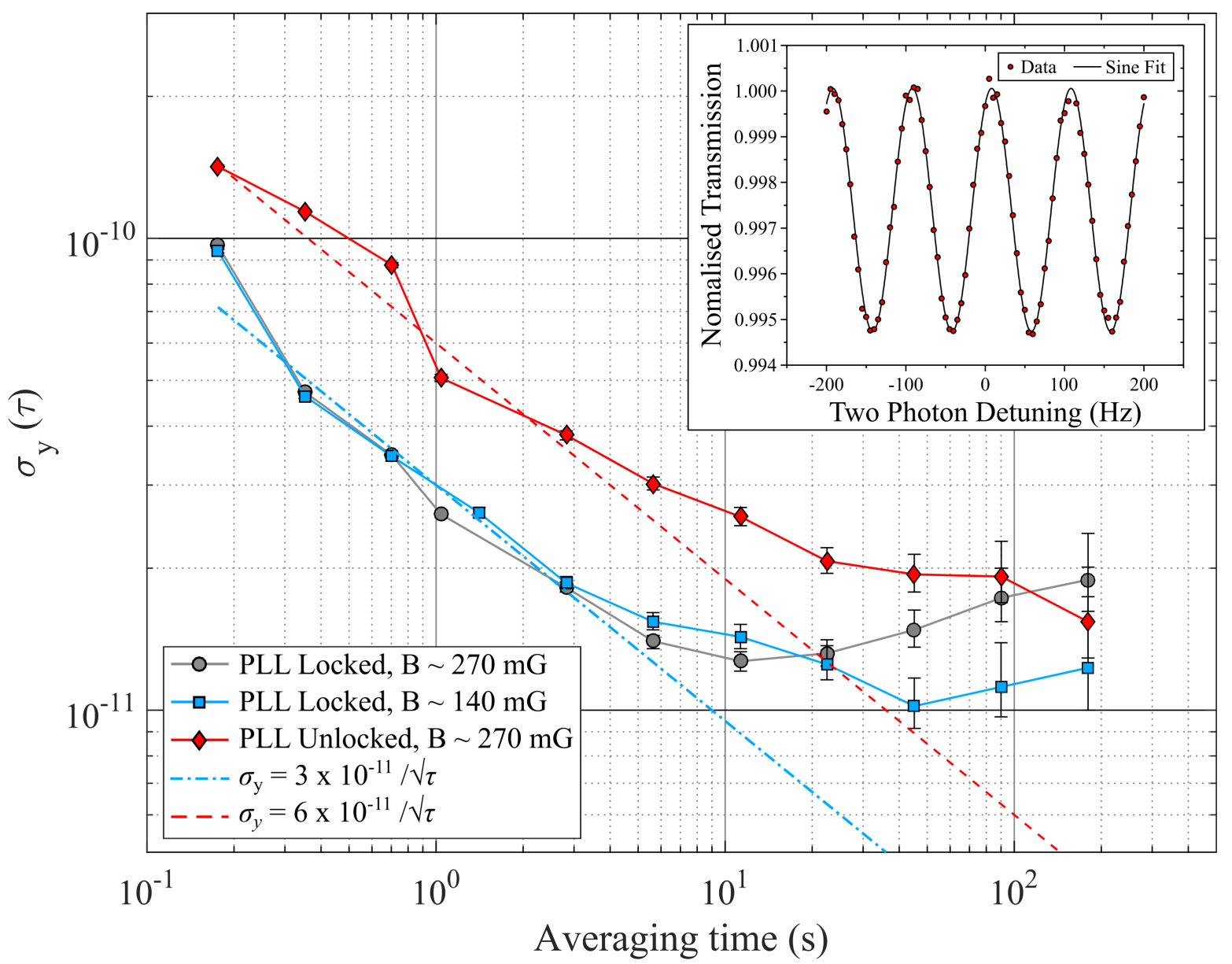

Figure 3. Measurements of the frequency stability of our apparatus in three configurations. Experimental data is shown with a free evolution time of $10 \mathrm{~ms}$ for CPT bias fields $B \sim 270 \mathrm{mG}$ with the PLL locked (grey circles); $B \sim 270 \mathrm{mG}$ with the PLL unlocked (red diamonds) and $B \sim 140 \mathrm{mG}$ with the PLL locked (blue squares). Additional straight lines are plotted to show the $1 / \sqrt{\tau}$ coefficients for the PLL locked and unlocked. The total CPT power reaching the atoms was $30 \mu \mathrm{W}$ and no steering from the Ramsey fringes is applied to the oscillator. (Inset) a typical $\mathrm{T}=10 \mathrm{~ms}$ Ramsey fringe obtained in our apparatus. The transmission was integrated for $28 \mu \mathrm{s}$ and $100 \mu \mathrm{s}$ for the detection and normalization regions described at the end of section 3. The data has been normalized again in post processing so that the peak of the Ramsey fringes corresponds to a transmission of 1.

In the short term, we plan to focus on upgrading our apparatus to improve the mid-term stability past the current floor near $1 \times 10^{-11}$. We hope to accomplish this by retro-reflecting the CPT beam to suppress Doppler shifts ${ }^{11}$ and by adding a magnetic shield to suppress the influence of magnetic field noise. In the long term, we plan to leverage the relative simplicity of the GMOT to develop a compact, transportable cold-atom CPT clock.

\section{Acknowledgment}

The authors gratefully acknowledge Leo Hollberg for useful technical discussions. We also acknowledge financial support from EPSRC (EP/M013294/1), the UK Quantum Technology Hub for Sensors and Metrology and the National Physical Laboratory. The research data supporting this publication can be accessed at: https://doi.org/10.15129/20ce7ab7-d4ea-4049-ad18-f72804255312. 


\section{REFERENCES}

[1] Ascarrunz, F. G., Dudin, Y. O., Delgado Aramburo, M. C., Savory, J., and Jefferts, S. R., "Long-Term Frequency Instability of a Portable Cold ${ }^{87} \mathrm{Rb}$ Atomic Clock," in [Proceedings of the 49th Annual Precise Time and Time Interval Systems and Applications Meeting], 107-110 (Jan. 2018).

[2] Pelle, B., Desruelle, B., Szmuk, R., and Holleville, D., "Cold-Atom-Based Commercial Microwave Clock at the $10^{-15}$ Level," in [2017 Joint Conference of the European Frequency and Time Forum and IEEE International Frequency Control Symposium (EFTF/IFCS)], 479-480 (Jul. 2017).

[3] Hendricks, R. J., Ozimek, F., Szymaniec, K., Nagórny, B., Dunst, P., Nawrocki, J., Beattie, S., Jian, B., and Gibble, K., "Cs Fountain Clocks for Commercial Realisations - an Improved and Robust Design," IEEE T. Ultra. Ferr. (Oct. 2018). Preprint.

[4] Vanier, J., "Atomic Clocks Based on Coherent Population Trapping: A Review," Appl. Phys. B 81, 421-442 (Aug. 2005).

[5] Shah, V. and Kitching, J., "Chapter 2 - Advances in Coherent Population Trapping for Atomic Clocks," in [Advances In Atomic, Molecular, and Optical Physics], Arimondo, E., Berman, P. R., and Lin, C. C., eds., 59, 21-74, Academic Press (Jan. 2010).

[6] Danet, J.-M., Kozlova, O., Yun, P., Guérandel, S., and de Clercq, E., "Compact Atomic Clock Prototype Based on Coherent Population Trapping," EPJ Web of Conferences 77, 00017 (Aug. 2014).

[7] Yun, P., Tricot, F., Calosso, C. E., Micalizio, S., François, B., Boudot, R., Guérandel, S., and de Clercq, E., "High-Performance Coherent Population Trapping Clock with Polarization Modulation," Phys. Rev. Appl. 7, 014018 (Jan. 2017).

[8] Abdel Hafiz, M., Coget, G., Yun, P., Guérandel, S., de Clercq, E., and Boudot, R., "A High-Performance Raman-Ramsey Cs Vapor Cell Atomic Clock," J. Appl. Phys. 121, 104903 (Mar. 2017).

[9] Liu, X., Ivanov, E., Yudin, V. I., Kitching, J., and Donley, E. A., "Low-Drift Coherent Population Trapping Clock Based on Laser-Cooled Atoms and High-Coherence Excitation Fields," Phys. Rev. Appl. 8, 054001 (Nov. 2017).

[10] Chen, X., Yang, G.-Q., Wang, J., and Zhan, M.-S., "Coherent Population Trapping-Ramsey Interference in Cold Atoms," Chin. Phys. Lett. 27, 113201 (Nov 2010).

[11] Esnault, F.-X., Blanshan, E., Ivanov, E. N., Scholten, R. E., Kitching, J., and Donley, E. A., "Cold-atom Double- $\Lambda$ Coherent Population Trapping Clock," Phys. Rev. A 88, 042120 (Oct. 2013).

[12] Blanshan, E., Rochester, S. M., Donley, E. A., and Kitching, J., "Light Shifts in a Pulsed Cold-Atom Coherent-Population-Trapping Clock," Phys. Rev. A 91, 041401 (Apr. 2015).

[13] Liu, X., Yudin, V. I., Taichenachev, A. V., Kitching, J., and Donley, E. A., "High Contrast Dark Resonances in a Cold-Atom Clock Probed with Counter-Propagating Circularly Polarized beams," Appl. Phys. Lett. 111, 224-102 (Nov. 2017).

[14] Pollock, J. W., Yudin, V. I., Shuker, M., Basalaev, M. Y., Taichenachev, A. V., Liu, X., Kitching, J., and Donley, E. A., "AC-Stark Shifts of Dark Resonances Probed with Ramsey Spectroscopy," Phys. Rev. A 98, 053424 (Nov. 2018).

[15] McGilligan, J. P., Micro-fabricated Diffractive Optics for Quantum Sensors and Atomic Clocks, PhD thesis, University of Strathclyde (Aug 2017).

[16] Elvin, R., Hoth, G. W., Wright, M. W., McGilligan, J. P., Arnold, A. S., Griffin, P. F., and Riis, E., "Raman-Ramsey CPT with a Grating Magneto-Optical Trap," in [2018 European Frequency and Time Forum (EFTF)], 61-64 (Apr. 2018).

[17] Santarelli, G., Laurent, P., Lemonde, P., Clairon, A., Mann, A. G., Chang, S., Luiten, A., and Salomon, C., "Quantum Projection Noise in an Atomic Fountain: A High Stability Cesium Frequency Standard," Phys. Rev. Lett. 82, 4619-4622 (Jun. 1999).

[18] Ludlow, A. D., Boyd, M. M., Ye, J., Peik, E., and Schmidt, P. O., "Optical Atomic Clocks," Rev. Mod. Phys. 87, 637-701 (Jun. 2015).

[19] Blanshan, E., Development of a Compact Cold-Atom Atomic Clock Based on Coherent Population Trapping, $\mathrm{PhD}$ thesis, University of Colorado, Boulder (Jan. 2014). 
[20] Nshii, C. C., Vangeleyn, M., Cotter, J. P., Griffin, P. F., Hinds, E. A., Ironside, C. N., See, P., Sinclair, A. G., Riis, E., and Arnold, A. S., "A Surface-Patterned Chip as a Strong Source of Ultracold Atoms for Quantum Technologies," Nat. Nanotechnol. 8, 321-324 (May 2013).

[21] McGilligan, J. P., Griffin, P. F., Elvin, R., Ingleby, S. J., Riis, E., and Arnold, A. S., "Grating Chips for Quantum Technologies," Sci. Rep. 7, 384 (Mar. 2017).

[22] Monroe, C., Swann, W., Robinson, H., and Wieman, C., "Very Cold Trapped Atoms in a Vapor Cell," Phys. Rev. Lett. 65, 1571-1574 (Sept. 1990).

[23] Zanon, T., Guérandel, S., de Clercq, E., Holleville, D., Dimarcq, N., and Clairon, A., "High Contrast Ramsey Fringes with Coherent-Population-Trapping Pulses in a Double Lambda Atomic System," Phys. Rev. Lett. 94, 193002 (May 2005).

[24] Kozlova, O., Danet, J.-M., Guérandel, S., and de Clercq, E., "Limitations of Long-Term Stability in a Coherent Population Trapping Cs Clock," IEEE T. Instr. Meas. 63, 1863-1870 (Jul. 2014).

[25] Rakholia, A. V., McGuinness, H. J., and Biedermann, G. W., "Dual-Axis High-Data-Rate Atom Interferometer via Cold Ensemble Exchange," Phys. Rev. Appl. 2, 054012 (Nov. 2014).

[26] Dick, G. J., "Local Oscillator Induced Instabilities in Trapped Ion Frequency standards," in [Proc. 19th Annual PTTI Application and Planning Meeting], 133-147 (1987).

[27] Danet, J.-M., Lours, M., Guérandel, S., and de Clercq, E., "Dick Effect in a Pulsed Atomic Clock Using Coherent Population Trapping," IEEE T Ultrason. Ferr. 61, 567-574 (Apr. 2014).

[28] Santarelli, G., Audoin, C., Makdissi, A., Laurent, P., Dick, G. J., and Clairon, A., "Frequency Stability Degradation of an Oscillator Slaved to a Periodically Interrogated Atomic Resonator," IEEE T Ultrason. Ferr. 45, 887-894 (Jul. 1998).

[29] McGuinness, H. J., Rakholia, A. V., and Biedermann, G. W., "High Data-Rate Atom Interferometer for Measuring Acceleration," Appl. Phys. Lett. 100, 011106-011106-4 (Jan. 2012).

[30] Dalton, B. J. and Knight, P. L., "Coherent Population Trapping and the Effect of Laser Phase Fluctuations," in [Lecture Notes in Physics], Harvey J.D., W. D., ed., 182, 213-225, Springer, Berlin, Heidelberg (2006).

[31] Warren, Z., Shahriar, M. S., Tripathi, R., and Pati, G. S., "Experimental and Theoretical Comparison of Different Optical Excitation Schemes for a Compact Coherent Population Trapping Rb Vapor Clock," Metrologia 54, 418 (June 2017). 